\title{
Analysis of Exhaled Breath during Surgery
}

\author{
J.Langejuergen $^{1}$, T.Hopmeier ${ }^{1}$, S.Meinen ${ }^{1}$, E.Carstens ${ }^{2}$, G.Theilmeier ${ }^{2}$, W.Koppert ${ }^{2}$, S.Zimmermann ${ }^{1}$ \\ ${ }^{1}$ Institute of Electrical Engineering and Measurement Technology, Leibniz University Hannover, \\ ${ }^{2}$ Deptartment of Anesthesiology and Intensive Care, Hannover Medical School, Germany, \\ Langejuergen@geml.uni-hannover.de
}

\begin{abstract}
:
The analysis of human breath is a non-invasive method to examine the physiological state of a patient by monitoring the exhaled metabolites. Here, we present a method to quantify ultra-low concentrations (ppt) of endogenous volatile organic compounds (VOCs) present in human breath after a short defined event like the administration of medication. The required high sensitivity is achieved by using atmospheric pressure ionization (API) in combination with a time-of-flight mass spectrometer (ToFMS) providing sufficient mass resolution to identify relevant endogenous VOCs in breath. At present, exhaled breath is sampled in the operating room for a few seconds on thermal desorption tubes and analyzed afterwards with our API-ToF-MS laboratory prototype. Long-term focus of the project is to miniaturize the system for bed-side online breath analysis. Furthermore, high resolving power allows identifying relevant biomarkers in the first place before developing miniaturized bed-side systems or even low-cost chemical sensors.
\end{abstract}

Key words: exhaled breath, ultra-low concentrations, atmospheric pressure ionization, endogenous volatile organic compounds, mass spectrometry

\section{Motivation}

The analysis of exhaled breath is a noninvasive method to examine the physiological state of a patient by monitoring the exhaled metabolites. Some analytical methods for breath gas analysis, such as GC-MS, GC-IMS, PTR-MS and SIFT-MS, as well as possible applications are described in [1]. The analytical challenge is to combine ultra-high sensitivity in order to detect even smallest concentrations of VOCs (ppt-range) and ultra-high resolving power giving a maximum of selectivity, to identify certain metabolites within the very complex matrix of exhaled breath. Furthermore, in order to investigate the patients' response to a certain time-discrete event, e.g. the administration of medication, a number of breath gas samples needs to be collected within a few minutes after the event giving just a small volume of e.g. $100 \mathrm{sccm}$ per breath sample to be analyzed. Thus, the exhaled metabolites are not pre-concentrated on the thermal desorption tube requiring ultra-high sensitivity. Here we present a method to collect pre- and post-event breath samples of ventilated patients and analyze these samples by API-ToF-MS. Long-term focus of the project is to miniaturize the system for online bed-side exhaled breath analysis. Furthermore, high resolving power allows identifying relevant biomarkers in the first place before developing miniaturized bed-side systems or even low-cost chemical sensors.

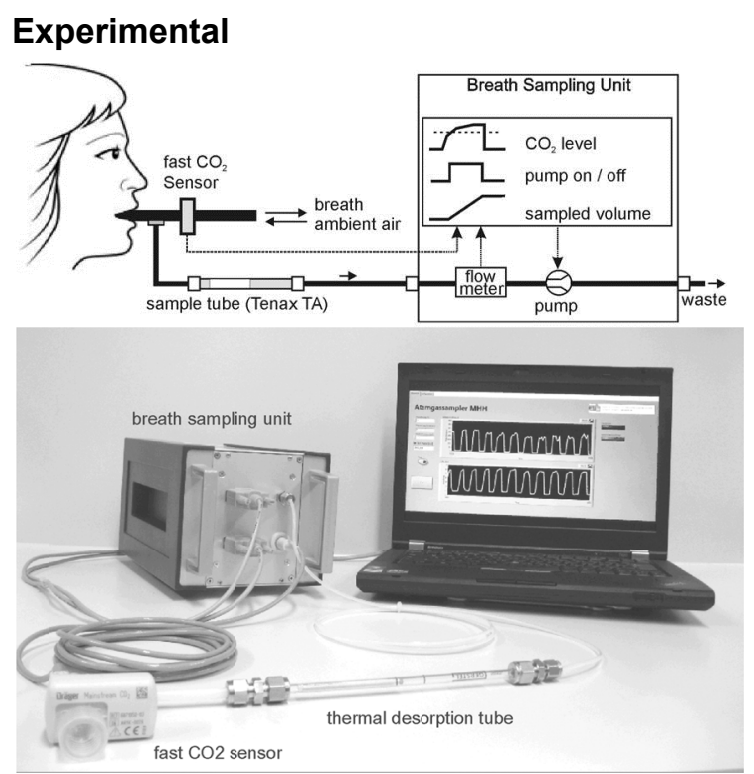

Fig. 1. Schematic setup of the breath sampling unit (top). An adjustable portion of the exhaled breath is pumped through a thermal desorption tube when the $\mathrm{CO}_{2}$ concentration of the exhaled breath exceeds $4 \%$. Thus, only end-tidal breath is sampled. The sampling unit is portable and is controlled via laptop (bottom).

In general, small portable systems are necessary for clinical bed-side applications. 
Since our measurement equipment is not miniaturized yet, the exhaled breath samples are collected by our breath-sampling-unit (fig. 1) and analyzed later in our laboratory. The $\mathrm{CO}_{2}$ concentration within the mainstream of the ventilation system is monitored by a fast $\mathrm{CO}_{2}$ sensor (Dräger, mainstream $\mathrm{CO}_{2}$ ). An adjustable portion of e.g. $100 \mathrm{sccm}$ of the exhaled breath is collected when the $\mathrm{CO}_{2}$ concentration exceeds a certain trigger level, here $4 \%$. This guarantees that only end-tidal breath is sampled. During surgery multiple samples are taken before and after a defined time-discrete event like the administration of medication. The VOCs present in the collected breath adsorb within a thermal desorption tube (Gerstel, OD $6 \mathrm{~mm}$, glass) filled with Tenax TA $(60 / 80)$. These tubes are then heated up $\left(200^{\circ} \mathrm{C}\right)$ in the laboratory (fig. 2) using a Gerstel TDS3 and the containing VOCs evaporate and are analyzed. The actual analysis of the exhaled breath sample is performed by a Bruker micrOTOF II time-offlight mass spectrometer. Both positive and negative ion spectra can be investigated. To obtain the necessary sensitivity we modified the MS by coupling our atmospheric pressure ionization source (API) to the inlet. Due to soft chemical ionization, as described in detail in [2], an almost fragment-free, easy to interpret mass spectrum and ultra-low detection limits (ppqrange) can be achieved. If necessary, a collision-induced fragmentation is possible. Furthermore, the hexapole RF-generator is changed in order to achieve the highest possible ion transfer rate for molecules of $30 \mathrm{u}$ to $300 \mathrm{u}$. The mass resolution is about 3000 for masses around $60 \mathrm{u}$. However, owing to concurring ionization processes a preseparation of the sampled VOCs by gas chromatography (GC) prior to ionization is necessary yet. We are using a GC (column: Restek, RTX volatiles, $30 \mathrm{~m}$, ID $530 \mu \mathrm{m}$ ) with a cold injection system (Gerstel KAS4) for preseparation. The initial temperature of the cryo trap of the cold injection system is $-150^{\circ} \mathrm{C}$. This is rapidly heated up $(16 \mathrm{~K} / \mathrm{s})$ for an injection into the GC.

One of our current projects is to optimize ionization parameters such as pressure and electric field as well as the amount of initially generated ions for a direct sample injection without pre-separation to allow online analysis.

\section{Results and Discussion}

In [3] we published preliminary results of the API-ToF-MS system demonstrating ultra low detection limits in the ppq-range with a direct sample gas injection. Most substances initially show no fragmentation and only protonated monomers $\left(\mathrm{M}+\mathrm{H}^{+}\right)$and for higher concentrations protonated dimers $\left(2 \mathrm{M}+\mathrm{H}^{+}\right)$are visible (figure 3, right). However, when necessary a collision-induced fragmentation within the MS ion interface is possible. For better visualization the results of breath gas analysis are displayed in form of heat-maps (fig. 3 , left). The ion mass (x-axis) is plotted versus the corresponding $\mathrm{GC}$ retention time (y-axis). The color indicates the absolute abundance. Masses within a range of $1 \mathrm{u}$ are summed up to simplify visual interpretation. For an automated analysis the full mass resolution can be used. For proof of concept, simple measurements are performed by sampling end-tidal breath of nonintubated volunteers and the inhaled air. Here, the difference of both heat-maps is shown to visualize the change in concentration. Acetone monomer and dimer are marked as an example for known endogenous substances. The acetone concentration in exhaled breath is about eight times higher than the concentration within the inhaled air (fig. 3, right). Within the exhaled breath of the non-intubated volunteers, there were a total of about 140 substances present at significantly higher concentrations then in the inhaled air. However, most of them were not identified yet.

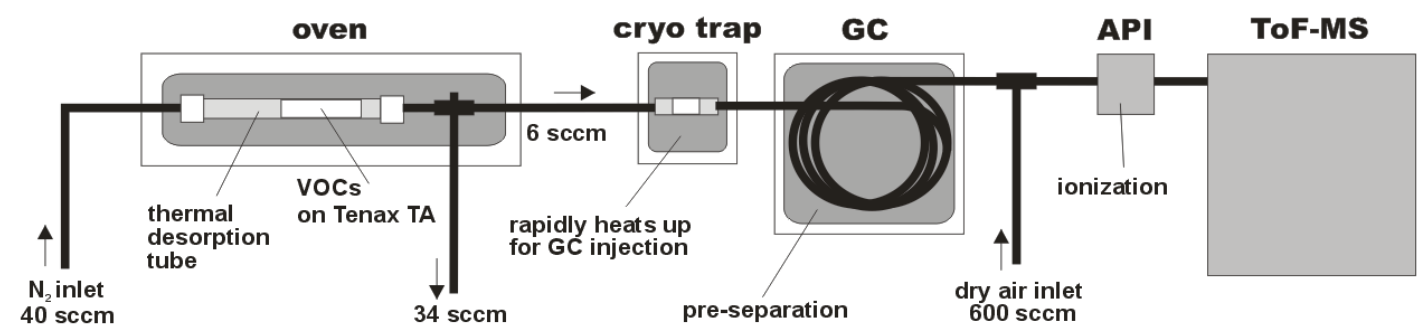

Fig. 2. Experimental setup for an analysis of the adsorbed volatile organic compounds. The thermal desorption tubes filled with Tenax TA are heated up (Gerstel TDS3, $200^{\circ} \mathrm{C}$ ), the containing VOCs evaporate and are transported with the gas flow into a cryo trap (Gerstel KAS4, $\left.-150^{\circ} \mathrm{C}\right)$. For a GC injection the trap is rapidly heated up $(16 \mathrm{~K} / \mathrm{s})$. The GC is necessary order to avoid concurring ionization processes between the different VOCs. The actual analysis of the exhaled breath is performed by a time-of-flight mass spectrometer (modified Bruker micrOToF II) coupled to our atmospheric pressure ionization source. 

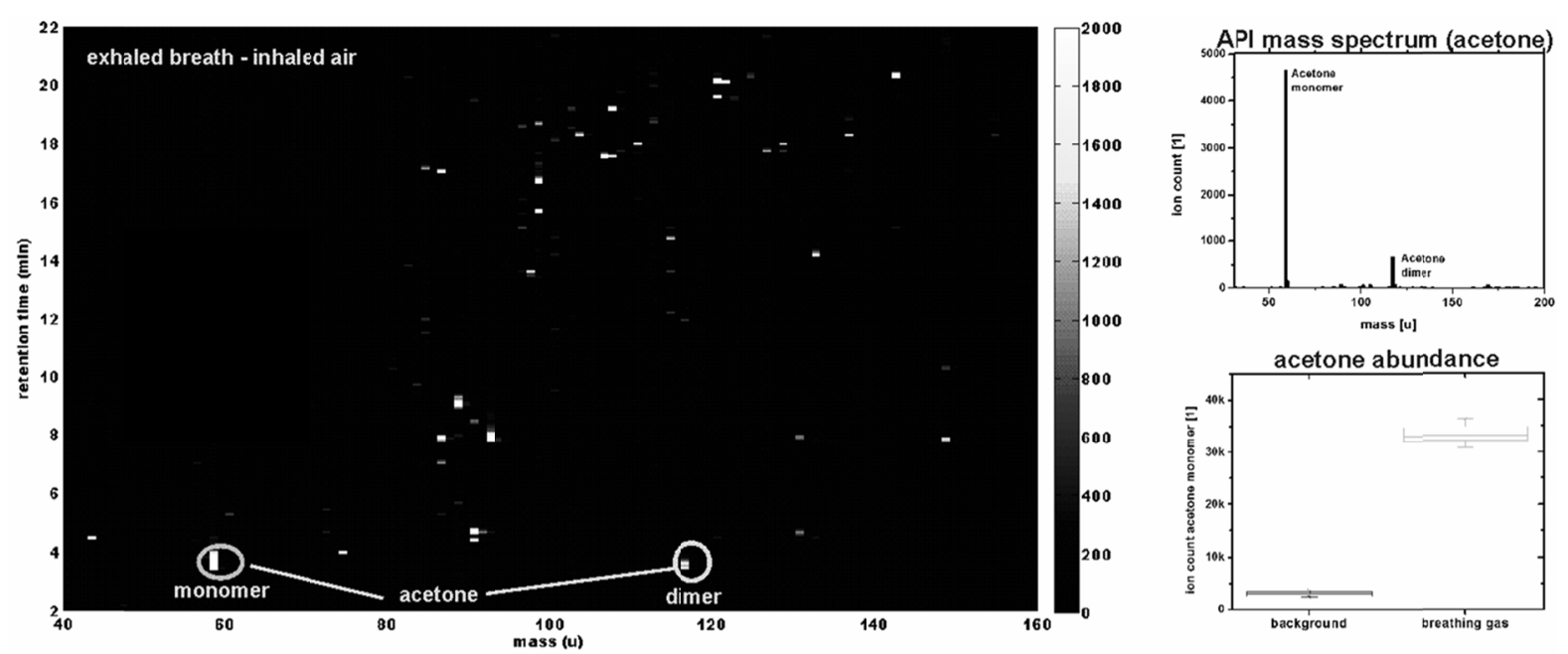

Fig. 3. The difference between inhaled air and exhaled breath of non-intubated volunteers is displayed as heatmap (left). The ion mass ( $x$-axis) is plotted versus the GC-retention time ( $y$-axis). The color indicates the absolute abundance. The mass spectrum and the abundance of acetone are shown (right). Due to the soft ionization process only the protonated monomer and dimer are visible. The acetone concentration in exhaled breath is about eight times higher than the concentration within the inhaled air.

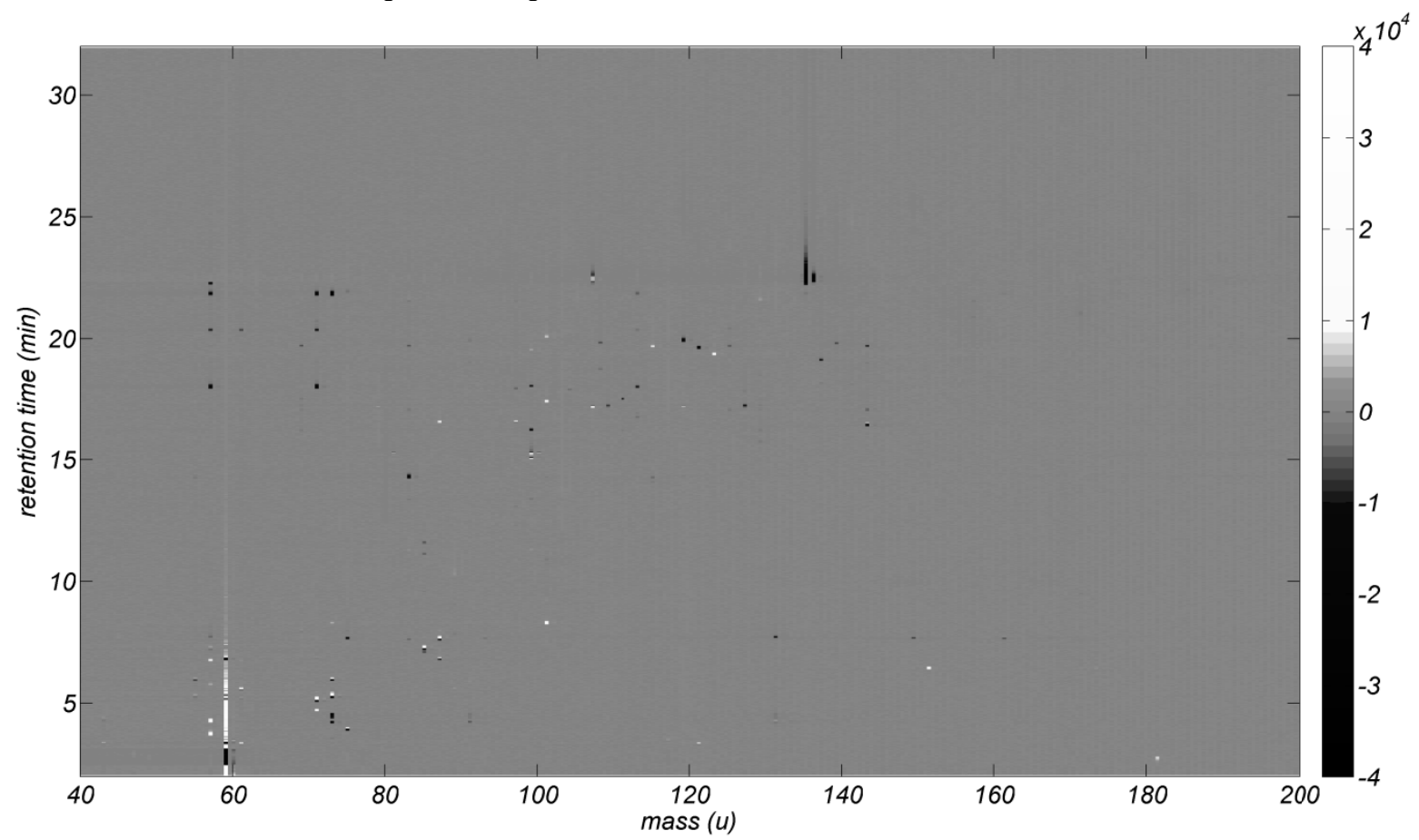

Fig. 4. The change in VOCs present in the exhaled breath of intubated patients during surgery is displayed as heat-map. The difference in spectra of one minute before and ten minutes after a defined' event are shown for a single patient. A bright color indicates an increase in concentration of a certain compound; a dark color indicates a decrease.

For the measurements performed with the intubated patients, the number of substances present in the sampled exhaled breath is increased compared to those of the nonintubated volunteers. Furthermore, some substances appear in significantly higher concentrations. This is due to the various medications administered prior to or during the introduction of anesthesia. As an example, the intravenously administered hypnotic agent propofol was found in the sampled breath. To identify the impact of a defined time-discrete event during the surgery, like the administration of a certain medication, the spectra generated before and after this event are subtracted and displayed as heat-map. As an example, the difference in spectra taken one minute before and ten minutes after a certain event is shown. (fig. 4). Thus, an increase or decrease in concentration of certain substances is visible. Both increase and decrease can be caused by the defined event as well as by drugs 
administered prior to the event, the individual metabolism, and state of health of the patient and are therefore individually different. Thus, in order to identify the specific response to a certain event, the change in spectra for multiple patients is compared. First results indicate several substances, most of them not identified yet, that show a comparable trend in the majority of the measurements. Some of these substances, e.g. propofol (fig. 5), show a decrease in concentration not correlated to the defined event. This is due to a continuous decrease in blood concentration of the medication given prior to or during the introduction of anaesthesia. Subject of recent research is to identify those substances that show a comparable trend for the majority the measured patients and correlate with the defined event.

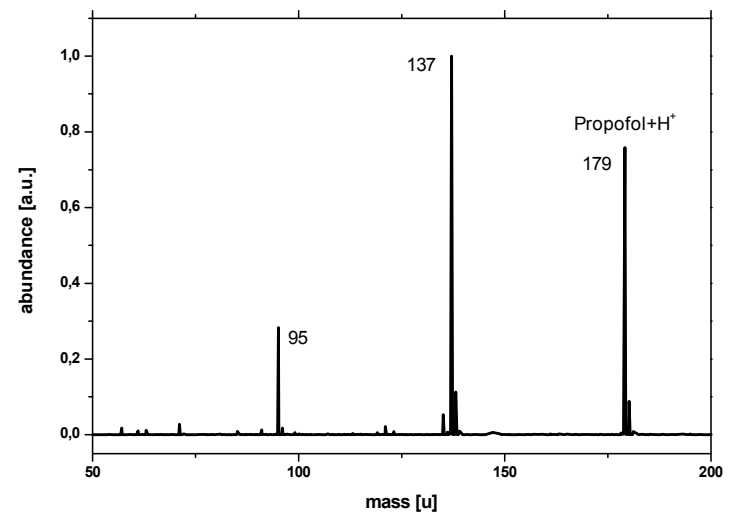

Fig. 5 Mass spectrum of propofol found in the sampled exhaled breath of intubated patients.

\section{Conclusion}

Here we present a method to sample end-tidal breath of intubated patients during surgery. In order to investigate the patients' response to a certain time-discrete event, a number of breath gas samples are collected within a short sampling time prior to and after the event. The samples are collected using thermal desorption tubes and analyzed in our laboratory with a mass spectrometer coupled to an atmospheric pressure ionization (API) source. Thus, even ultra-low concentrations of volatile organic compounds present in the sampled breath can be detected and distinguished. However, in order to avoid concurring ionization processes within the API source, the sampled breath is pre-separated by a gas chromatograph. The resulting spectra are visualized in form of heatmaps where the mass of the detected ions is plotted versus the GC-retention time and the absolute abundance. VOCs of endogenous origin like acetone are detected as well as traces of exogenous substances like propofol. In order to identify the impact of a defined event, the differences in spectra of the exhaled breath prior to and after the event are compared for every patient. First results indicate several substances, most of them not identified yet, that show a comparable trend in the majority of the measurements. Subject of recent research is to identify relevant substances and correlate them to the defined event. Long-term focus of the project is to miniaturize the system for bed-side online breath analysis.

\section{References}

[1] A. Amann, D. Smith, Breath Analysis for Clinical Diagnosis and Therapeutic Monitoring, World Scientific Publishing, 2005.

[2] G.A. Eiceman, Z. Karpas, Ion Mobility Spectrometry, CRC Press, 2005.

[3] J. Langejuergen, P. Cochems, S. Zimmermann (2011): A Simple Atmospheric Pressure Ionization Source Coupled to a Mass Spectrometer for Breath Analysis, 10. Dresdner Sensor Symposium 